\title{
openheart Effects of dietary fats on blood lipids: a review of direct comparison trials
}

\author{
James J DiNicolantonio, James H O’Keefe
}

To cite: DiNicolantonio JJ, O'Keefe JH. Effects of dietary fats on blood lipids: a review of direct comparison trials. Open Heart 2018;5:e000871. doi:10.1136/ openhrt-2018-000871

Accepted 3 July 2018

Check for updates

(c) Author(s) (or their employer(s)) 2018. Re-use permitted under CC BY-NC. No commercial re-use. See rights and permissions. Published by BMJ.

Preventive Cardiology, Saint Lukes Mid America Heart Institute, Kansas City, Missouri, USA

Correspondence to Dr James J DiNicolantonio; jjdinicol@gmail.com

\section{INTRODUCTION}

Saturated fat has been demonised as a dietary culprit in heart disease due to its ability to raise low-density lipoprotein cholesterol (LDL-C), whereas omega-6 polyunsaturated fatty acid (PUFA) has been regarded as heart healthy due to its ability to lower total and LDL-C. And replacing saturated fat with omega- 6 has consistently been found to lower total cholesterol and LDL-C levels. ${ }^{12}$ This has been the cornerstone for the belief that the omega-6 PUFA linoleic acid is heart healthy. However, the changes in LDL-C do not take into account the overall changes in the entire lipoprotein profile. For example, saturated fat appears to decrease small-dense LDL (sdLDL) and increase large buoyant LDL. ${ }^{3}$ As a high concentration of sdLDL and a low concentration of large buoyant LDL is associated with an increased risk of coronary heart disease (CHD), ${ }^{4}$ saturated fat may not necessarily increase the risk of CHD. Furthermore, compared with LDL-C, sdLDL (and oxidised LDL) seem to have a greater impact on atherosclerosis and cardiovascular disease. ${ }^{5}$

While saturated fat (particularly lauric acid) has been shown to increase total and LDL-C, there is also an increase in high-density lipoprotein cholesterol (HDL-C). ${ }^{6}$ Moreover, unsaturated fatty acids have a less prominent effect for increasing HDL-C compared with saturated fat. Thus, it is hard to interpret the overall risk of consuming foods high in saturated fat versus omega-6 PUFA when the former may improve sdLDL and HDL-C, whereas the latter may lower LDL-C but increases LDL susceptibility to oxidation and may lower HDL-C. ${ }^{78}$ Indeed, high concentrations of HDL-C are associated with greater protection from coronary artery disease and other cardiovascular diseases. ${ }^{9} 10$ Thus, the overall effect on the lipoprotein profile must be considered when assessing cardiovascular risk with dietary fats and fatty acids.

The effects of replacing saturated fat with omega-6 PUFA on triglycerides (TG), very LDL (VLDL) and HDL are also inconsistent. ${ }^{11}$ Thus, it is impossible to know what the overall health impact is when saturated fat is replaced with omega-6 PUFA.

\section{MONOUNSATURATED FAT VERSUS SATURATED FAT}

Monounsaturated fat ((MUFA) such as oleic acid, which is found in olive oil, has classically been thought of as being heart healthy as olive oil is the main dietary fat used in the Mediterranean region, which is well known for its low risk for cardiovascular disease.

Meals high in both MUFA and saturated fat lead to what's called 'postprandial lipaemia' or a rise in chylomicrons, chylomicron remnants and triglycerides, which are thought to be harmful to the arteries. One study showed that compared with saturated fat, oleic acid causes a greater secretion of chylomicrons that are larger in size and contain greater amounts of TGs. ${ }^{12}$ Chylomicrons are the largest of the lipoproteins and they carry exogenous dietary TGs, whereas VLDL carries TGs from the liver. Despite a higher peak, postprandial TG content with the consumption of olive oil, adhering to a Mediterranean diet results in a faster decrease in TG levels. In other words, the postprandial rise in TGs returns to baseline levels quicker with a high MUFA (oleic acid) test meal compared with a meal high in saturated fat. ${ }^{13}$ Thus, while the peak TG level may be higher with olive oil, the level drops faster with oleic acid. This may partially explain some of the health benefits of MUFA versus saturated fat.

The increased clearance of TGs from the blood with the consumption of oleic acid is likely the result of the formation of larger sized chylomicrons compared with smaller more 'VLDL-sized' chylomicrons from saturated fat. This suggests that consuming a diet high in butter and cream, and low in MUFA, may increase the risk of chylomicrons penetrating into the endothelium, as smaller particles are more likely to penetrate, which 
Box 1 The benefits of a high-monounsaturated fatty acid meal (eg, oleic acid, olive oil) versus a high-saturated fat meal (such as cream/butter)

Larger (less atherogenic) chylomicrons/very low-density lipoprotein (LDL) particles.

- Reduced postprandial lipaemia (faster clearance of postprandial lipids).

- Greater reduction in fasting triglyceride levels.

- Reduction in coagulation factors (factor VIIc and factor VIla).

- Increased fat oxidation rate (greater fat burning and less fat storage).

A greater shift from small-dense LDL to large buoyant LDL.

then become stuck under the endothelium, oxidise and can lead to atherosclerosis. Another study showed that compared with a meal high in saturated fat, a MUFA-rich meal leads to a greater fat oxidation rate at 3 and 4 hours postdose, as well as lower blood coagulation factors, such as factor VIIc and factor VIIa, suggesting that MUFAs may promote less weight gain and a reduction in the risk for thrombosis versus saturated fat. ${ }^{14}$ Similar results were found in another study where the consumption of MUFA lead to a lower postprandial rise in coagulation factors versus saturated fat. ${ }^{12}$

In a 4-week trial in 38 healthy volunteers, an oleic acid-enriched diet as compared with a saturated fat diet reduced factor VII; however, this benefit was not found with an omega-6-enriched diet. ${ }^{15}$ In another study, compared with diets rich in saturated fat (lauric or palmitic acid), diets rich in oleic acid reduced factor VII again, whereas plasminogen activator inhibitor-1 (PAI1 ), which is an inhibitor of the fibrinolytic system, was higher in diets rich in palmitic acid versus oleic acid and lauric acid. ${ }^{16}$ Finally, a high MUFA Mediterranean Diet has been found to increase flow-mediated dilation and reduce LDL, apolipoprotein $\mathrm{B}$ and P-selectin compared with a baseline diet high in saturated fat. ${ }^{17}$ These studies suggest that MUFA, as compared with saturated fat, improves the lipid profile and the hypercoagulable state. The benefits of a meal rich in oleic acid versus saturated fat are summarised in box 1 .

\section{OMEGA-3 VERSUS OMEGA-6}

The optimal $n-6 / n-3$ ratio in the UK Diet (OPTILIP) was a randomised parallel design trial in 258 patients aged $45-70 .{ }^{18}$ OPTILIP compared four diets providing approximately $6 \%$ energy as PUFAs but with varying dietary omega-6/omega-3 ratios (3-5:1 compared with 10:1, which was the control diet). The three omega-3 enriched diets contained (1) alpha-linolenic acid (ALA), (2) eicosapentaenoic acid (EPA) and docosahexaenoic acid (DHA) or (3) both. Compared with the high omega-6/omega-3 ratio diet, which ended up being around 11:1, the diet enriched in EPA/DHA (providing an omega- $6 /$ omega-3 ratio of around $3: 1$ ) caused a reduction in fasting and postprandial TGs as well as sdLDL. Reducing the omega-6/omega-3 ratio by giving
EPA/DHA also reduced VLDL, increased LDL particle size and increased HDL2. These benefits were not found with ALA. Moreover, increasing the intake of linoleic acid from $4.7 \%$ to $7 \%$ energy reduced the protective HDL2 $(35.2 \%$ vs $31.7 \%){ }^{18}$

LIPGENE was a multicentre, randomised controlled trial in Europe testing four isocaloric diets in 99 patients with the metabolic syndrome. ${ }^{19}$ The diets consisted of (1) a high-fat (38\% energy) saturated fat-rich diet; (2) a high-fat MUFA-enriched diet, (3) a low-fat (28\% energy) high-complex carbohydrate diet (that also contained $1.24 \mathrm{~g} /$ day of high oleic sunflower oil) and (4) the same low-fat diet but with an additional $1.24 \mathrm{~g}$ /day of longchain omega-3 PUFAs for 12 weeks. LDL phenotype B (sdLDL) shifted to phenotype A (large-buoyant LDL) in both the high-fat MUFA-enriched diet and the low-fat long-chain omega-3 diet. The LDL density was also reduced in these groups. The frequency of LDL phenotype B was lowered by $37 \%$ in the long-chain omega-3 PUFA group and by $15 \%$ in the high-fat MUFA rich diet. TGs were also lowered in both of these groups, which may have partially explained the results. Opposite effects were found in both the high-saturated fat diet as well as the low-fat, high-oleic sunflower oil diet, which increased LDL density, despite the latter lowering total cholesterol. The LIPGENE study concluded, 'This study demonstrates the efficacy of dietary omega-3 PUFA to modify proatherogenic to less atherogenic LDL phenotype in patients with metabolic syndrome'. ${ }^{19}$

Considering that LDL particle size predicts cardiovascular risk and progression of $\mathrm{CHD},{ }^{20} 21$ omega- 6 vegetable oils reducing LDL particle size may increase the risk of CHD and ischaemic stroke. ${ }^{22}$ This is because sdLDL particles are more susceptible to oxidation. Indeed, sdLDL particles remain out in the blood longer due to a reduced affinity for the LDL receptors in the liver and also more readily cross the subendothelial space to oxidise and cause atherosclerotic plaque formation. ${ }^{23}$

The heart healthy properties of marine omega-3s have been questioned due to their ability to increase LDL-C, however their ability to transform atherogenic smalldense LDL (pattern B) to large-buoyant LDL (pattern A) likely outweighs any harm with a higher LDL-C level. The increase in large buoyant LDL with marine omega-3 may be due to an increased lipoprotein lipase gene expression in plasma. ${ }^{24}$

The effect of omega-3 PUFAs on the susceptibility of LDL oxidation is controversial. Some studies suggest that marine omega-3s, provided as fish, do not increase oxidised LDL with decreases being noted in urinary isoprostane excretion. ${ }^{25}{ }^{26}$ Plasma $\mathrm{F}(2)$-isoprostane and malondialdehyde, which are markers of oxidative stress, were significantly lower with fish oil supplementation versus sunflower oil. ${ }^{27}$ Maximal rates of phosphatidylcholine hydroperoxide and cholesteryl linoleate hydroperoxide formation were also significantly lower with fish oil (3.4 g of EPA/DHA/day) compared with safflower oil (providing $10.5 \mathrm{~g}$ of linoleic acid per day). The authors 
concluded that 'supplementation of postmenopausal women with fish oil does not increase overall oxidation of LDL ex vivo compared with dietary oils rich in oleate and linoleate'. ${ }^{28}$

In a randomised double-blind cross-over study in familial combined hyperlipidaemia giving $3.4 \mathrm{~g}$ of EPA/ DHA (as Omacor) for 8 weeks significantly lowered plasma TGs and VLDL (27\% and $18 \%$, respectively). ${ }^{29}$ Despite an increase in LDL-C levels by $21 \%$, there was an increase in the more buoyant, fast floating LDL-1 and LDL-2 with a decrease in the denser, slower floating LDL-3 subclass. This study confirmed the findings that EPA/ DHA can increase LDL-C but at the same time reduces LDL density. The average LDL size was not significantly reduced with fish oil, but this was thought to be due to the baseline LDL size (25.0) already being quite low. ${ }^{29}$ Thus, the marine omega-3 fats EPA/DHA are associated with slight increases in LDL-C, which may be because DHA downregulates the LDL-receptor, possibly decreasing LDL clearance.$^{30}$ However, DHA seems to increase LDL size and buoyancy, which indicates less atherogenic LDL.

Another double-blind parallel design placebo-controlled trial in 42 adults found that $4 \mathrm{~g}$ /day of EPA/ DHA for 12 weeks increased LDL-C by $13 \%(\mathrm{p}<0.0001)$. However, there were increases in both large (LDL1 $(+2.2 \mathrm{mg} / \mathrm{dL})$ and LDL2 $(+2.6 \mathrm{mg} / \mathrm{dL}))$ and sdLDL $(\mathrm{LDL} 3(+6.3 \mathrm{mg} / \mathrm{dL})$ and LDL4 $(+0.04 \mathrm{mg} / \mathrm{dL})) .{ }^{31}$ The changes for LDL1-3 were all statistically significant except for the change in LDL4. The authors concluded that 'iIn this population of hypertriglyceridemic adults, dietary supplementation with fish oil resulted in an increase in total LDL-C which was distributed relatively evenly across the range of smaller and more atherogenic as well as larger and less atherogenic LDL particles'. ${ }^{31}$

Another study in 57 men with dyslipidaemia were randomly assigned to one of the three diets enriched with flaxseed oil (providing around $25 \mathrm{~g}$ of ALA per day), sunflower oil (providing around $25 \mathrm{~g}$ of linoleic acid per day) or sunflower oil plus fish oil (providing around $3 \mathrm{~g}$ of EPA/DHA per day) for 12 weeks. ${ }^{32}$ All three diets reduced cholesterol levels. Only the flax and fish oil groups reduced TG levels, which was most pronounced with the fish oil group $(-23 \%, \mathrm{p}<0.001)$. Moreover, the fish oil group had a significant reduction in smalldense LDL $(-22 \%, p=0.003)$ and a significant increase in HDL2. Additionally, only the fish oil group had a significant reduction in the TC/HDL ratio. which is a better predictor of CHD compared with LDL-C. Both the flax oil and sunflower oil groups caused a decrease in HDL $(-10.5 \%$ and $-5.6 \%$, respectively), whereas the group given fish oil had a slight increase in HDL $(+3 \%)$. The proportion of sdLDL decreased in all groups, but this was only significant in the fish oil supplemented diet. A shift in the LDL subclasses towards larger lighter LDL particles was found with the group supplemented with fish oil. The reductions in TGs and sdLDL that occurred after fish oil consumption correlated with an increase in membrane DHA levels, suggesting that if DHA levels
Box 2 Benefits of maintaining a low omega-6/omega-3 ratio on blood lipids

Reduction in fasting and postprandial triglycerides.

- Reduction in small-dense low-density lipoprotein.

- Increase in protective high-density lipoprotein 2.

are not increased small-dense LDL may not be reduced. The authors concluded, 'in conclusion, fish-oil produced predictable changes in plasma lipids and small-dense LDL that were not reproduced by the ALA-enriched diet' ${ }^{32}$

Thus, the overall evidence suggests that marine omega-3s reduce small-dense LDL, which are more atherogenic and hence supplementing with marine omega-3s in order to shift a small-dense LDL pattern to a larger more buoyant LDL particle pattern will likely reduce the risk of cardiovascular disease. Indeed, at least seven randomised controlled trials have found that omega-3 fatty acids increase LDL particle size or shift LDL particle distribution from atherogenic small-dense LDL particles (pattern B) to large buoyant particles (pattern A) ${ }^{33}$ The benefit of supplementing with marine omega-3s likely depends on whether someone has patter B LDL to begin with and if triglyceride levels are significantly reduced.

The benefits of maintaining a low omega- $6 / 3$ ratio and the harms of omega- 6 PUFA on the lipid profile are covered in boxs 2 and 3, respectively.

\section{DHA VERSUS EPA ON BLOOD LIPIDS}

The improvements in LDL density with the consumption of marine omega-3s seem to be largely from DHA, which, when compared with EPA, increases LDL particle size $^{34}$ and reduces sdLDL particles. ${ }^{35}$ However, supplementation with purified EPA has also been found to reduce sdLDL, remnant lipoproteins and lower inflammation (C-reactive protein) in patients with metabolic syndrome. ${ }^{19}{ }^{36}$ Thus, DHA may simply be better than EPA at providing beneficial changes in LDL particle size and/ or density. In one study of 74 healthy normolipidaemic men and women $2.3 \mathrm{~g}$ of DHA per day, but not $2.2 \mathrm{~g}$ of EPA, significantly increased HDL levels by $13 \%$ (from 1.60 to $1.81 \mathrm{mmol} / \mathrm{L}$ or $28.8 \mathrm{mg} / \mathrm{dL}$ to $32.58 \mathrm{mg} / \mathrm{dL}$ ). ${ }^{33}$ This may have been due to the twofold greater reduction in fasting triacylglycerol with DHA versus EPA.

Another group of authors concluded that despite DHA being found in lower concentrations compared with EPA in many supplements, DHA has 'equally important antiarrhythmic, antithrombotic and antiatherogenic effects'. ${ }^{37}$ One randomised study of 38 dyslipidaemic

\section{Box 3 Harms of omega- 6 on blood lipids}

- No change or increase in small-dense low-density lipoprotein.

- No change or decrease in protective high- density lipoprotein 2.

- Little change in triglycerides. 
patients showed that DHA ( $3 \mathrm{~g} /$ day) reduced TG levels more than EPA (3 g/day) as compared with placebo ( $\mathrm{p}=0.006 \mathrm{vs} \mathrm{p}=0.026$, respectively) ${ }^{38}$ Both omega-3 fatty acids improved systemic arterial compliance.

DHA has been found to have a greater effect at reducing TGs compared with $\mathrm{EPA}^{39}$ and this may be why DHA has been more consistently found to increase LDL particle size. ${ }^{34}$ In one study, despite DHA increasing LDL-C by $8 \%(p=0.019)$, there was a highly significant increase in LDL particle size $(+0.25 \mathrm{~nm}, \mathrm{p}=0.002)$ and a significant increase in large HDL2 $(p=0.004)$, effects which are considered beneficial. The HDL raising effects of DHA has been confirmed in another direct comparison trial, where EPA significantly reduced apo-A1 $(p=0.0003) .{ }^{40}$ Two trials now suggest that EPA is not converted to DHA in humans (unless the EPA dose is extremely high); in fact, giving EPA may actually lower DHA in serum and platelet phospholipids. ${ }^{340}$ However, in these trials, DHA increased both DHA and EPA in serum and platelet phospholipids suggesting significant retroconversion of DHA to EPA in humans. One group of authors concluded that 'DHA is known to accumulate in the central nervous system and in cardiac tissue and advanced atherosclerotic plaques are enriched with more DHA than EPA after dietary supplementation...DHA is selectively incorporated into extracirculatory pools whereas EPA has priority in the circulatory pool'. ${ }^{40}$ Due to these two distinct differences between EPA and DHA, it is likely best to supplement with both omega-3s.

Besides decreasing sdLDL and postprandial TG levels, fish oil has been found to lower the concentrations of medium and small VLDLs, which may be more atherogenic compared with larger VLDL. ${ }^{41}{ }^{42}$ In fact, it has been suggested that if VLDL penetrates into the subendothelium, which is more likely to occur with smaller VLDL particles, it is even more atherogenic than LDL as it delivers a larger oxidative load. By reducing VLDL concentrations and increasing VLDL particle size, fish oil likely reduces the number of VLDL particles that penetrate and oxidise in the subendothelium. This benefit also likely occurs with LDL since fish oil increases LDL size and reduces its density. Finally, the Agency for Health Research and Quality systematic review found that in general omega-3s increase HDL by $3-5 \mathrm{mg} / \mathrm{dL},{ }^{43}$ whereas omega-6 PUFA decreases HDL. ${ }^{44}$ Boxs 4 and 5 summarise the beneficial effects of DHA and EPA on lipids, whereas box 6 summarises the benefits of DHA versus EPA.

Box 4 The beneficial effects of docosahexaenoic acid (DHA) on blood lipids

- Reduces trigyceride levels.

- Increases very low-density lipoprotein (LDL) size.

- Reduces small-dense LDL.

- Increases large buoyant LDL.

Increases high-density lipoprotein 2.
Box 5 The beneficial effects of eicosapentaenoic acid on blood lipids

Reduces triglycerides.

- Increases very low density lipoprotein (LDL) size.

- May reduce small-dense LDL.

\section{Box 6 Benefits of docosahexaenoic acid versus} eicosapentaenoic acid

Greater triglyceride lowering.

- Greater increase in large buoyant low-density lipoprotein (LDL) and greater reduction in small-dense LDL.

Greater rise in high density lipoprotein.

\section{CONCLUSION}

In summary, compared with a high-MUFA meal, such as olive oil, a high saturated fat meal from butter or cream seems to have worse overall effects on blood lipids. Despite the fact that omega-6 PUFA lowers LDL, it can also reduce LDL particle size and lower the protective HDL2 potentially increase the risk for CHD. The overall effect on blood lipids for marine omega-3 EPA and DHA seems to be protective due to overall improvements in LDL particle size and density as well as reductions in VLDL, TGs, and increases in HDL.

Contributors Both authors contributed to the final manuscript.

Funding The authors have not declared a specific grant for this research from any funding agency in the public, commercial or not-for-profit sectors.

Competing interests JJD is author of The Salt Fix. JHO is the Chief Medical Officer and Founder of CardioTabs, a nutraceutical company and does have a major ownership interest in the company. CardioTabs does sell products that contain omega-3.

Patient consent Not required.

Provenance and peer review Not commissioned; internally peer reviewed.

Open access This is an open access article distributed in accordance with the Creative Commons Attribution Non Commercial (CC BY-NC 4.0) license, which permits others to distribute, remix, adapt, build upon this work non-commercially, and license their derivative works on different terms, provided the original work is properly cited, appropriate credit is given, any changes made indicated, and the use is non-commercial. See: http://creativecommons.org/licenses/by-nc/4.0/.

\section{REFERENCES}

1. Shepherd J, Packard CJ, Grundy SM, et al. Effects of saturated and polyunsaturated fat diets on the chemical composition and metabolism of low density lipoproteins in man. J Lipid Res 1980;21:91-9.

2. Turner JD, Le NA, Brown WV. Effect of changing dietary fat saturation on low-density lipoprotein metabolism in man. Am J Physiol 1981;241:E57-E63.

3. Dreon DM, Fernstrom HA, Campos $\mathrm{H}$, et al. Change in dietary saturated fat intake is correlated with change in mass of large lowdensity-lipoprotein particles in men. Am J Clin Nutr 1998;67:828-36.

4. Griffin BA, Freeman DJ, Tait GW, et al. Role of plasma triglyceride in the regulation of plasma low density lipoprotein (LDL) subfractions: relative contribution of small, dense LDL to coronary heart disease risk. Atherosclerosis 1994;106:241-53.

5. Henriksen T, Mahoney EM, Steinberg D. Enhanced macrophage degradation of biologically modified low density lipoprotein. Arteriosclerosis 1983;3:149-59.

6. Mensink RP, Zock PL, Kester AD, et al. Effects of dietary fatty acids and carbohydrates on the ratio of serum total to HDL cholesterol and 
on serum lipids and apolipoproteins: a meta-analysis of 60 controlled trials. Am J Clin Nutr 2003;77:1146-55.

7. Reaven P, Parthasarathy S, Grasse BJ, et al. Effects of oleaterich and linoleate-rich diets on the susceptibility of low density lipoprotein to oxidative modification in mildly hypercholesterolemic subjects. J Clin Invest 1993;91:668-76.

8. Reaven PD, Grasse BJ, Tribble DL. Effects of linoleate-enriched and oleate-enriched diets in combination with alpha-tocopherol on the susceptibility of LDL and LDL subfractions to oxidative modification in humans. Arterioscler Thromb 1994;14:557-66.

9. Kitamura A, Iso H, Naito $Y$, et al. High-density lipoprotein cholesterol and premature coronary heart disease in urban Japanese men. Circulation 1994;89:2533-9.

10. Stein O, Stein Y. Atheroprotective mechanisms of HDL. Atherosclerosis 1999;144:285-301.

11. Weintraub MS, Zechner R, Brown A, et al. Dietary polyunsaturated fats of the W-6 and W-3 series reduce postprandial lipoprotein levels. Chronic and acute effects of fat saturation on postprandial lipoprotein metabolism. J Clin Invest 1988;82:1884-93.

12. Bergouignan A, Momken I, Schoeller DA, et al. Metabolic fate of saturated and monounsaturated dietary fats: the Mediterranean diet revisited from epidemiological evidence to cellular mechanisms. Prog Lipid Res 2009;48:128-47.

13. Tholstrup T, Sandström B, Bysted A, et al. Effect of 6 dietary fatty acids on the postprandial lipid profile, plasma fatty acids, lipoprotein lipase, and cholesterol ester transfer activities in healthy young men. Am J Clin Nutr 2001;73:198-208.

14. Silva KD, Kelly $C N$, Jones $A E$, et al. Chylomicron particle size and number, factor VII activation and dietary monounsaturated fatty acids. Atherosclerosis 2003;166:73-84.

15. Turpeinen AM, Mutanen M. Similar effects of diets high in oleic or linoleic acids on coagulation and fibrinolytic factors in healthy humans. Nutr Metab Cardiovasc Dis 1999;9:65-72.

16. Temme EH, Mensink RP, Hornstra G. Effects of diets enriched in lauric, palmitic or oleic acids on blood coagulation and fibrinolysis. Thromb Haemost 1999;81:259-63.

17. Fuentes F, López-Miranda J, Sánchez E, et al. Mediterranean and low-fat diets improve endothelial function in hypercholesterolemic men. Ann Intern Med 2001;134:1115-9.

18. Griffin MD, Sanders TA, Davies IG, et al. Effects of altering the ratio of dietary n-6 to n-3 fatty acids on insulin sensitivity, lipoprotein size and postprandial lipemia in men and postmenopausal women aged 45-70 y: the OPTILIP Study. Am J Clin Nutr 2006;84:1290-8.

19. Hartwich J, Malec MM, Partyka L, et al. The effect of the plasma $n-3 / n-6$ polyunsaturated fatty acid ratio on the dietary LDL phenotype transformation - insights from the LIPGENE study. Clin Nutr 2009;28:510-5.

20. Austin MA, Breslow JL, Hennekens $\mathrm{CH}$, et al. Low-density lipoprotein subclass patterns and risk of myocardial infarction. JAMA 1988;260:1917-21.

21. Rizzo M, Berneis K. Low-density lipoprotein size and cardiovascular risk assessment. QJM 2006;99:1-14.

22. Slowik A, Iskra T, Turaj W, et al. LDL phenotype B and other lipid abnormalities in patients with large vessel disease and small vessel disease. J Neurol Sci 2003;214:11-16.

23. Anber V, Griffin BA, McConnell M, et al. Influence of plasma lipid and LDL-subfraction profile on the interaction between low density lipoprotein with human arterial wall proteoglycans. Atherosclerosis 1996;124:261-71.

24. Khan S, Minihane AM, Talmud PJ, et al. Dietary long-chain n-3 PUFAs increase LPL gene expression in adipose tissue of subjects with an atherogenic lipoprotein phenotype. $J$ Lipid Res 2002;43:979-85.

25. Mori TA, Dunstan DW, Burke V, et al. Effect of dietary fish and exercise training on urinary $\mathrm{F} 2$-isoprostane excretion in non-insulindependent diabetic patients. Metabolism 1999;48:1402-8.

26. Mori TA, Puddey IB, Burke V, et al. Effect of omega 3 fatty acids on oxidative stress in humans: GC-MS measurement of urinary F2isoprostane excretion. Redox Rep 2000;5:45-6.
27. Higdon JV, Liu J, Du SH, Sh D, et al. Supplementation of postmenopausal women with fish oil rich in eicosapentaenoic acid and docosahexaenoic acid is not associated with greater in vivo lipid peroxidation compared with oils rich in oleate and linoleate as assessed by plasma malondialdehyde and $\mathrm{F}(2)$-isoprostanes. Am J Clin Nutr 2000;72:714-22.

28. Higdon JV, Du SH, Lee YS, et al. Supplementation of postmenopausal women with fish oil does not increase overall oxidation of LDL ex vivo compared to dietary oils rich in oleate and linoleate. J Lipid Res 2001;42:407-18.

29. Calabresi L, Donati D, Pazzucconi F, et al. Omacor in familial combined hyperlipidemia: effects on lipids and low density lipoprotein subclasses. Atherosclerosis 2000;148:387-96.

30. Sperling LS, Nelson JR. History and future of omega-3 fatty acids in cardiovascular disease. Curr Med Res Opin 2016;32:301-11.

31. Oelrich B, Dewell A, Gardner CD. Effect of fish oil supplementation on serum triglycerides, LDL cholesterol and LDL subfractions in hypertriglyceridemic adults. Nutr Metab Cardiovasc Dis 2013;23:350-7.

32. Wilkinson P, Leach C, Ah-Sing EE, et al. Influence of alpha-linolenic acid and fish-oil on markers of cardiovascular risk in subjects with an atherogenic lipoprotein phenotype. Atherosclerosis 2005;181:115-24.

33. Egert S, Kannenberg F, Somoza V, et al. Dietary alpha-linolenic acid, EPA, and DHA have differential effects on LDL fatty acid composition but similar effects on serum lipid profiles in normolipidemic humans. J Nutr 2009;139:861-8.

34. Mori TA, Burke V, Puddey IB, et al. Purified eicosapentaenoic and docosahexaenoic acids have differential effects on serum lipids and lipoproteins, LDL particle size, glucose, and insulin in mildly hyperlipidemic men. Am J Clin Nutr 2000;71:1085-94.

35. Kelley DS, Siegel D, Vemuri M, et al. Docosahexaenoic acid supplementation improves fasting and postprandial lipid profiles in hypertriglyceridemic men. Am J Clin Nutr 2007;86:324-33.

36. Satoh N, Shimatsu A, Kotani K, et al. Purified eicosapentaenoic acid reduces small dense LDL, remnant lipoprotein particles, and C-reactive protein in metabolic syndrome. Diabetes Care 2007;30:144-6.

37. Mori TA, Woodman RJ. The independent effects of eicosapentaenoic acid and docosahexaenoic acid on cardiovascular risk factors in humans. Curr Opin Clin Nutr Metab Care 2006;9:95-104.

38. Nestel P, Shige H, Pomeroy $\mathrm{S}$, et al. The $\mathrm{n}-3$ fatty acids eicosapentaenoic acid and docosahexaenoic acid increase systemic arterial compliance in humans. Am J Clin Nutr 2002;76:326-30.

39. Buckley R, Shewring B, Turner R, et al. Circulating triacylglycerol and apoE levels in response to EPA and docosahexaenoic acid supplementation in adult human subjects. Br J Nutr 2004;92:477-83.

40. Grimsgaard S, Bonaa KH, Hansen JB, et al. Highly purified eicosapentaenoic acid and docosahexaenoic acid in humans have similar triacylglycerol-lowering effects but divergent effects on serum fatty acids. Am J Clin Nutr 1997;66:649-59.

41. Li Z, Lamon-Fava S, Otvos J, et al. Fish consumption shifts lipoprotein subfractions to a less atherogenic pattern in humans. $J$ Nutr 2004;134:1724-8.

42. Schaefer EJ, Lichtenstein AH, Lamon-Fava S, et al. Effects of National Cholesterol Education Program Step 2 diets relatively high or relatively low in fish-derived fatty acids on plasma lipoproteins in middle-aged and elderly subjects. Am J Clin Nutr 1996;63:234-41.

43. Robinson JG, Stone NJ. Antiatherosclerotic and antithrombotic effects of omega-3 fatty acids. Am J Cardiol 2006;98:39-49.

44. Mensink RP, Katan MB. Effect of dietary fatty acids on serum lipids and lipoproteins. A meta-analysis of 27 trials. Arterioscler Thromb 1992;12:911-9.

45. Wooten JS, Biggerstaff KD, Ben-Ezra V. Responses of LDL and HDL particle size and distribution to omega-3 fatty acid supplementation and aerobic exercise. J Appl Physiol 2009;107:794-800. 